\section{Arkivoc \\ =}

Archive for

Organic Chemistry
The Free Internet Journal

for Organic Chemistry
Paper

Arkivoc 2017, part ii, 107-117

\title{
Diethyl boronobenzylphosphonates as substrates in Petasis reactions
}

\author{
Agata Rydzewska, Anna Olender, Artur Mucha, and Paweł Kafarski*
}

Department of Bioorganic Chemistry, Faculty of Chemistry, Wrocław University of Science and Technology, Wybrzeże Wyspiańskiego 27, 50-370 Wrocław, Poland

Email:pawel.kafarski@pwr.edu.pl

\section{Dedicated to Professor Jacek Młochowski on the occasion of his $80^{\text {th }}$ anniversary}

Received 06-06-2016

Accepted 08-09-2016

Published on line $08-16-2016$

\section{Abstract}

The Petasis reaction of $\alpha$-( $N$-benzylamino)boronobenzylphosphonates with amines and salicylaldehyde or glyoxalic acid gives the desired products in moderate to good yields. The efficiency of the reaction is strongly dependent on the structure of the amine.<smiles>[R]N[R]</smiles>
$48 \mathrm{~h} / \mathrm{rt} / \mathrm{N}_{2}$<smiles>[R]CO[Pb]C(NCc1ccccc1)c1ccc(C([R])N[R])cc1</smiles>

Keywords: Aminophosphonates, Petasis reaction, benzylboronates 


\section{Introduction}

Aminophosphonic acids were first considered simply as analogues of amino acids, in which the phosphonic acid moiety replaces the carboxylic function. By acting as antimetabolites of amino acids they exhibited a variety of physiological effects, being considered as possible novel drugs and agrochemicals. Further, especially when introduced into a peptide chain, they were recognized as compounds which mimic the tetrahedral transition state of enzymatic hydrolysis of esters and amides. Consequently, in most cases they had been designed and synthesized as inhibitors of important proteinases, the targets for potential drugs against a variety of diseases. Frequently such efforts appeared successful and resulted in compounds of variable and promising physiological activity and in commercially available substances.$^{1-5}$ In addition, aminophosphonic acids and their derivatives are able to form complexes with a variety of metal ions. ${ }^{6}$ These findings ensure that they still continue to attract wide interest.

There are a significant number of papers devoted to the synthesis of aminophosphonates. ${ }^{5,7-10}$ However, in most cases they give rise to compounds of relatively simple structure. Therefore, there is a strong need for elaboration of new synthetic procedures leading to functionalized aminophosphonates. The availability of diethyl $\alpha$-( $N$-benzylamino)boronobenzylphosphonates ${ }^{11,12}$ creates such a possibility by application of a Petasis reaction. In this paper we describe the scope and limitations of the use of these substrates.

The Petasis reaction, referred to also as the boronic acid Mannich reaction, is an extremely useful procedure providing molecules of complex architecture. ${ }^{13-15}$ It is a three-component reaction between amine, organoborate and aldehyde or ketone and proceeds via an imine with the organic ligand of the boronic acid acting as the nucleophile, like the role of the enolizable ketone component in the original Mannich reaction.

\section{Results and Discussion}

Diethyl $\alpha$-( $N$-benzylamino)-4-boronobenzylphosphonate 1 and $\alpha$-( $N$-benzylamino)-3-boronobenzylphosphonate $\mathbf{2}$ were prepared through classical addition of diethyl phosphite to the appropriate Schiff base obtained from commercially available formylphenylboronic acids (Scheme 1).

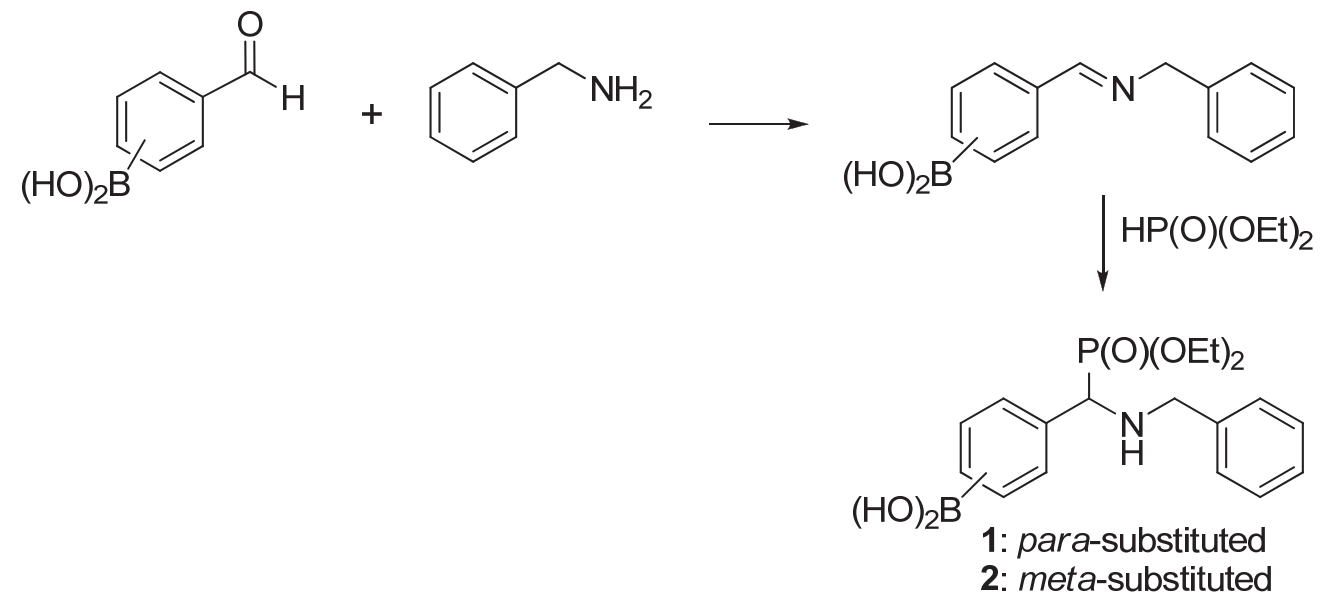

Scheme 1. Preparation of substrates for Petasis reaction.

Aminobenzylphosphonates have already been used as amine components in Petasis reactions with good results. ${ }^{16}$ Thus, compounds $\mathbf{1}$ and $\mathbf{2}$ might be expected to be the sources of both boronic and amino moieties. This assumption, tested when using compound $\mathbf{1}$ and salicylic aldehyde and glyoxalic acid as substrates, gave 
negative results despite application of varying ratios of reagents, two (toluene and ethanol) solvents and different temperatures. As a consequence, benzylboronic acids $\mathbf{1}$ and $\mathbf{2}$ were used as boronic reagents in further studies.

The course of the reaction was optimized by reacting compound $\mathbf{1}$, salicylic aldehyde and morpholine in reactions carried out for 48 hours at room temperature. As seen from Table 1, the best results were obtained when the reaction was carried out in dichloromethane and toluene. In boiling dichloromethane for $7 \mathrm{~h}$ the reaction gave the desired product in $47 \%$ isolated yield.

Table 1. Reaction of compound 1 with salicylic aldehyde and morpholine in various solvents

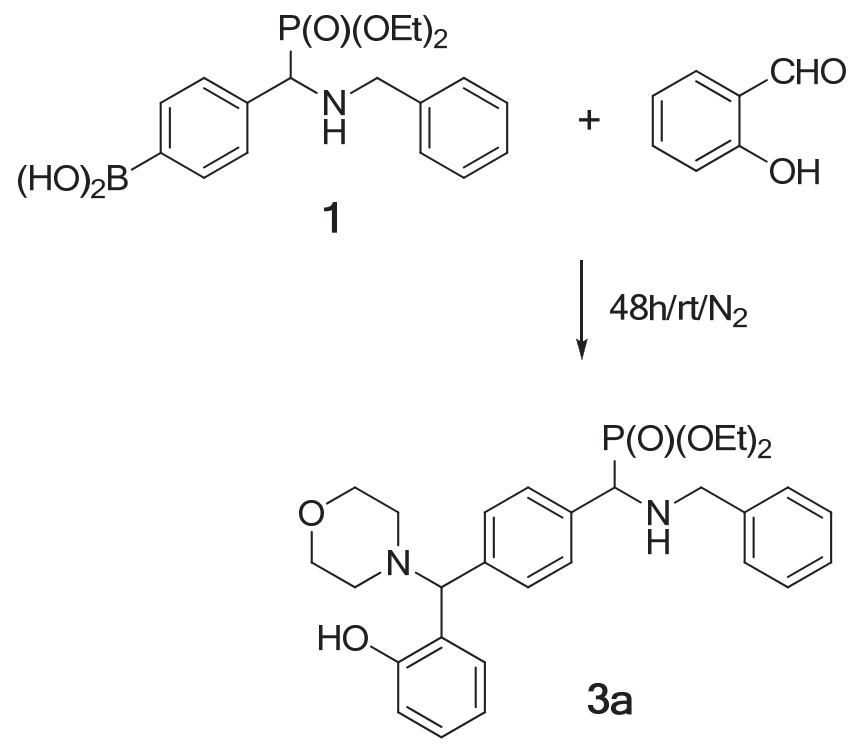

\begin{tabular}{ccc}
\hline Entry & Solvent & ${\text { Yield }(\%)^{\mathrm{a}}}^{\mathrm{a}}$ \\
\hline 1 & Dichloromethane & 53 \\
2 & Toluene & 44 \\
3 & 1,4-Dioxane & 35 \\
4 & Tetrahydrofuran & 22 \\
5 & Ethanol & 0 \\
6 & Methanol & 13 \\
\hline a estimated by ${ }^{31}$ P NMR
\end{tabular}

The use of piperidine and bis(dimethoxyethyl)amine as substrates also gave satisfactory results, when the reactions were carried out in boiling dichloromethane for $6 \mathrm{~h}$, and the corresponding products $3 \mathbf{b}$ and $3 \mathrm{c}$ (Scheme 2) were obtained in $96 \%$ and $69 \%$ yields respectively. The use of compound 2 as a substrate gave similar results and compounds $\mathbf{4 a}$ and $\mathbf{4 b}$ were obtained in $63 \%$ and $38 \%$ yields respectively. All the products were obtained as equimolar mixtures of diastereomers.

Some more detailed studies were carried out using glyoxalic acid as substrate. The results of these studies are collected in Table 2. As can be seen from the Table, the Petasis reaction is quite capricious and its course unpredictable. Thus, it lacks universal value and is applicable only for selected substrates. In the case of this substrate, ethanol and acetonitrile appeared to be solvents of choice. As seen from the ${ }^{31} \mathrm{P} N \mathrm{NMR}$ spectra this reaction once more yields equimolar mixtures of diastereoisomeric products. 
<smiles>CCOC(=O)C(NCc1ccccc1)c1ccc(C(c2ccccc2O)N2CCCCC2)cc1</smiles><smiles>CCOC(=O)C(NCc1ccccc1)c1ccc(C(c2ccccc2O)N(CCOC)CCOC)cc1</smiles><smiles>CCOC(OCC)C(NCc1ccccc1)c1cccc(C(c2ccccc2O)N2CCOCC2)c1</smiles>

$4 a$<smiles>CCOC(OCC)C(NCc1ccccc1)c1cccc(C(c2ccccc2O)N2CCCCC2)c1</smiles>

$4 b$

Scheme 2. Products obtained by reacting piperidine and bis(dimethoxyethyl)amine with compounds $\mathbf{1}$ and $\mathbf{2}$ and salicylic aldehyde.

Some additional features of this reaction require comment. First, we observed that compounds $\mathbf{1}$ and $\mathbf{2}$ are unstable at elevated temperatures and so prolonged heating leads to cleavage of the carbon-to-boron bond. Second, we expected that in the reaction with diethanolamine formation of its boronic ester would accompany the process (this reagent is commonly used to protect a boronic acid). Surprisingly, this was not the case.

Finally, we tried using a protected form of substrate $\mathbf{1}$ - its pinacol ester $\mathbf{7}$. This compound was obtained in a one-pot procedure, by reacting 4-formylphenylboronic acid with pinacol, followed by formation of the Schiff base with benzylamine and then the addition of diethyl phosphite (Scheme 3).<smiles>CC(C)(O)C(C)(C)O</smiles><smiles>CC1(C)OB(c2ccc(C=O)cc2)OC1(C)C</smiles><smiles>NCc1ccccc1</smiles><smiles>CCOC(NCc1ccccc1)c1ccc(B2OC(C)(C)C(C)(C)O2)cc1</smiles><smiles>CCOCC</smiles><smiles>CC1(C)OB(c2ccc(/C=N/Cc3ccccc3)cc2)OC1(C)C</smiles>

Scheme 3. Preparation of compound 7. 
Table 2. Reaction of compounds 1 and $\mathbf{2}$ with glyoxalic acid and amines<smiles>[R]N[I+][Na]</smiles>

1 or 2 $\downarrow 48 \mathrm{~h} / \mathrm{rt} / \mathrm{N}_{2}$<smiles>[R7]NC([R])c1ccc(C(NCc2ccccc2)C(=O)O[R])cc1</smiles>

\begin{tabular}{|c|c|c|c|c|}
\hline Compound & Substrate & $\begin{array}{l}\text { Amine } \\
\mathrm{R}^{1}-\mathrm{N}-\mathrm{R}^{2}\end{array}$ & Reaction conditions & $\begin{array}{l}\text { Yield } \\
(\%)^{\mathrm{a}}\end{array}$ \\
\hline $5 a$ & 1 & morpholine & ethanol, room temp, $48 \mathrm{~h}$ & 59 \\
\hline \multirow[t]{2}{*}{$5 b$} & & piperidine & $\begin{array}{l}\text { 96\% ethanol, } 10 \mathrm{~h} \text { under } \\
\text { reflux }\end{array}$ & 25 \\
\hline & & & $\mathrm{CH}_{3} \mathrm{CN}, 6.5 \mathrm{~h}$ under reflux & 37 \\
\hline $5 c$ & & bis(dimethoxyethyl)amine & $\mathrm{CH}_{3} \mathrm{CN}, 3.5 \mathrm{~h}$ under reflux & 57 \\
\hline \multirow[t]{2}{*}{$5 d$} & & benzylamine & $\begin{array}{c}\text { dichloromethane, room } \\
\text { temp, } 48 \mathrm{~h}\end{array}$ & 3 \\
\hline & & & $\begin{array}{c}\text { ethanol, room temp, } 48 \mathrm{~h} \\
\text { dichloromethane or }\end{array}$ & 0 \\
\hline $5 e$ & & diisopropylamine & $\begin{array}{c}\text { ethanol or } 1,4 \text {-dioxane, } \\
7 \mathrm{~h} \text { under reflux }\end{array}$ & 0 \\
\hline $5 f$ & & diethanloamine & $\begin{array}{l}96 \% \text { ethanol, } 10 \mathrm{~h} \text { under } \\
\text { reflux }\end{array}$ & 0 \\
\hline $6 a$ & 2 & morpholine & $\begin{array}{l}\text { 96\% ethanol, } 7 \mathrm{~h} \text { under } \\
\text { reflux }\end{array}$ & 32 \\
\hline $6 b$ & & piperidine & $\begin{array}{c}96 \% \text { ethanol, } 13.5 \mathrm{~h} \\
\text { under reflux }\end{array}$ & 23 \\
\hline $6 c$ & & bis(dimethoxyethyl)amine & $\begin{array}{l}96 \% \text { ethanol, } 10 \mathrm{~h} \text { under } \\
\text { reflux }\end{array}$ & 0 \\
\hline
\end{tabular}

${ }^{a}$ Isolated yields

Despite of the use of various solvents (ethanol, acetonitrile, dichloromethane and toluene) and various reaction conditions we failed to obtain products of condensation of compound 7 with glyoxalic acid and benzylamine. In the case of morpholine, when the reaction was carried out in ethanol ( $24 \mathrm{~h}$ at room temperature followed by $7 \mathrm{~h}$ at reflux) the desired product was obtained in $11 \%$ yield (based on ${ }^{31} \mathrm{P} N M R$ ). Thus, this substrate is far less useful than the unprotected boronic acid. 


\section{Conclusion}

We report the application of diethyl $\alpha$-( $N$-benzylamino)boronobenzylphosphonates as a substrates in Petasis reaction. Optimization of reaction conditions when applying salicylic aldehyde and glyoxalic acid have shown that this reaction is capricious and its course is mainly dependent on the structure of the amine used, with morpholine and piperidine being the best substrates. Our studies indicate that aminophosphonic acids functionalized with boronic moiety in aromatic ring could be considered as substrates in Petasis reaction although each set of substrates requires individual optimization of reaction conditions.

\section{Experimental Section}

General. ${ }^{1} \mathrm{H}$ NMR, ${ }^{13} \mathrm{C}$ NMR, ${ }^{31} \mathrm{P}$ NMR and ${ }^{11} \mathrm{~B}$ NMR were recorded on a Brüker DRX $300 \mathrm{MHz}\left({ }^{1} \mathrm{H}: 300.13 \mathrm{MHz}\right.$, $\left.{ }^{31} \mathrm{P}: 121.51 \mathrm{MHz}\right)$ and Brüker Avance II Ultrashield Plus $600 \mathrm{MHz}\left({ }^{1} \mathrm{H}: 600.58 \mathrm{MHz},{ }^{31} \mathrm{P}: 243.21 \mathrm{MHz},{ }^{13} \mathrm{C}: 151.03\right.$ $\mathrm{MHz}$ and $\left.{ }^{11} \mathrm{~B}: 192.69 \mathrm{MHz}\right)$ using internal references or solvent peaks as reference. The chemical shifts $(\delta)$ and coupling constants $(J)$ are expressed in ppm and Hertz respectively. The signal assignments were made using HSCQ spectra. Electrospray mass spectra were recorded at Faculty of Chemistry, Wroclaw University of Technology using Waters LCT Premier XE mass spectrometer. Flash chromatography was carried out using Interchim Puriflash 430evo system and Puriflash colums with silica gel beads of the diameter of 15 or $30 \mu \mathrm{m}$. Reagents and starting materials were directly used as obtained commercially.

Diethyl $\boldsymbol{\alpha}$-( $\mathrm{N}$-benzylamino)boronobenzylphosphonates - general procedure. To the suspension of formylphenylboronic acid $(3.0 \mathrm{~g}, 20 \mathrm{mmol})$ in DCM $(50 \mathrm{~mL})$ was added benzylamine $(2.24 \mathrm{~mL}, 20 \mathrm{mmol})$ and the mixture was heated with azeotropic removal of the formed water for $1.5 \mathrm{~h}$. Evaporation of the solvents gave a glassy solid which was dissolved in toluene $(60 \mathrm{~mL})$. To this solution diethyl phosphite $(2.68 \mathrm{~mL}, 20$ mmol) was added and the mixture refluxed for $3 \mathrm{~h}$. After evaporation of solvents the residue was chromatographed using flash system

Diethyl $\boldsymbol{\alpha}$-( $\boldsymbol{N}$-benzylamino)-4-boronobenzylphosphonate (1) was obtained, after chromatographic purification using $\mathrm{CHCl}_{3} / \mathrm{MeOH}$ gradient (from $100 \%$ to $85 \%$ of $\mathrm{CHCl}_{3}$ ) as eluent, as yellow solidifying oil $(6.0 \mathrm{~g}, 79 \%) .{ }^{31} \mathrm{P}$ NMR (MeOD, $243 \mathrm{MHz}$ ): $\delta_{\mathrm{P}} 23,78 .{ }^{11} \mathrm{~B}$ NMR (MeOD, $193 \mathrm{MHz}$ ): $\delta_{\mathrm{B}}$ 28.29. ${ }^{1} \mathrm{H}$ NMR (MeOD, $600 \mathrm{MHz}$ ): $\delta_{\mathrm{H}} 1.12$ and 1.29 (each: $t, J_{\mathrm{HH}} 7.1 \mathrm{~Hz}, 3 \mathrm{H}, \mathrm{OCH}_{2} \mathrm{CH}_{3}$ ), 3.52 and 3.80 (each: d, $J_{\mathrm{HH}} 13,3 \mathrm{~Hz}, 2 \mathrm{H}, \mathrm{NHCH}_{2}$ ), 3.81-3.83 and 3.90-3.97 (each: $\left.\mathrm{m}, 2 \mathrm{H}, \mathrm{OCH}_{2} \mathrm{CH}_{3}\right), 4.03\left(\mathrm{~d},{ }^{2} J_{\mathrm{HP}} 20.8 \mathrm{~Hz}, 1 \mathrm{H}, \mathrm{CHP}\right), 4.06-4.13\left(\mathrm{~m}, 2 \mathrm{H}, \mathrm{OCH}_{2} \mathrm{CH}_{3}\right), 7.21-7.26(\mathrm{~m}$, $3 \mathrm{H}, \mathrm{Ar}), 7.29-7.31(\mathrm{~m}, 2 \mathrm{H}, \mathrm{Ar}), 7.41$ (bs, $2 \mathrm{H}, \mathrm{Ar}), 7.68$, (bs, $1 \mathrm{H}, \mathrm{Ar}), 7.817 .41$ (bs, $1 \mathrm{H}, \mathrm{Ar}) .{ }^{13} \mathrm{C}$ NMR (MeOD, 151 $\mathrm{MHz}$ ): $\delta_{\mathrm{C}} 16.52$ and 16.72 (each: $\mathrm{d}, J_{\mathrm{CP}} 6.0 \mathrm{~Hz}, \mathrm{OCH}_{2} \mathrm{CH}_{3}$ ), 51.91 (d, $J_{\mathrm{CP}} 18.1 \mathrm{~Hz}, \mathrm{CH}_{2} \mathrm{Ph}$ ), 60.14 (d, $J_{\mathrm{CP}} 155.5 \mathrm{~Hz}$, CHP), 64.26 (d, J $\left.J_{\mathrm{CP}} 7.6 \mathrm{~Hz}, \mathrm{OCH}_{2} \mathrm{CH}_{3}\right), 64,45\left(\mathrm{~d},{ }^{2} J_{\mathrm{CP}} 6.0 \mathrm{~Hz}, \mathrm{OCH}_{2} \mathrm{CH}_{3}\right), 128.21$ (s, Ar), $128.82(\mathrm{~s}, \mathrm{Ar}), 129.12$ (d, J 6.0 Hz, Ar), 129.38 (s, Ar), 129.50 (s, Ar), 135.05 (s, Ar), 140.36 (s, Ar). Upon recording of MS in methanol this compound forms monomethyl ester (confirmed independently by ${ }^{1} \mathrm{H}$ NMR), HRMS (ESI-TOF) $m / z$ [MOMe+H ${ }^{+}$], Calcd. for $\mathrm{C}_{19} \mathrm{H}_{28} \mathrm{NO}_{5} \mathrm{BP}$ : 392.1798, found 392.1801.

Diethyl $\boldsymbol{\alpha}$-( $\boldsymbol{N}$-benzylamino)-3-boronobenzylphosphonate (2) was obtained, after chromatographic purification using $\mathrm{CHCl}_{3} / \mathrm{MeOH}$ gradient (from $100 \%$ to $85 \%$ of $\mathrm{CHCl}_{3}$ ) as eluent, as yellow solidifying oil $(6.0 \mathrm{~g}, 79 \%) .{ }^{31} \mathrm{P}$ NMR (MeOD, $243 \mathrm{MHz}$ ): $\delta_{\mathrm{p}} 23,99$ and 24,12. ${ }^{11} \mathrm{~B} \mathrm{NMR}$ (MeOD, $193 \mathrm{MHz}$ ): $\delta_{\mathrm{B}} 28.00 .{ }^{1} \mathrm{H} \mathrm{NMR}$ (MeOD, $600 \mathrm{MHz}$ ): $\delta_{\mathrm{H}} 1.13$ and 1.30 (each: t, $J_{\mathrm{HH}} 7.1 \mathrm{~Hz}, 3 \mathrm{H}, \mathrm{OCH}_{2} \mathrm{CH}_{3}$ ), 3.53 and 3.82 (each: $\mathrm{d}, \mathrm{J}_{\mathrm{HH}} 13.3 \mathrm{~Hz}, 1 \mathrm{H}, \mathrm{CH}_{2} \mathrm{Ph}$ ), 3.82-3.85 and 3.92-398 (each : $\mathrm{m}, 2 \mathrm{H}, \mathrm{OCH}_{2} \mathrm{CH}_{3}$ ), $4.03\left(\mathrm{~d}, \mathrm{~J}_{\mathrm{HP}} 20.5 \mathrm{~Hz}, 1 \mathrm{H}, \mathrm{CHP}\right.$ ), 4.06-4.14 (m, $\left.2 \mathrm{H}, \mathrm{OCH}_{2} \mathrm{CH}_{3}\right), 7.22-7.29$ $(\mathrm{m}, 3 \mathrm{H}, \mathrm{Ar}), 7.31-7.36(\mathrm{~m}, 2 \mathrm{H}, \mathrm{Ar}), 7.39-7.48(\mathrm{~m}, 2 \mathrm{H}, \mathrm{Ar}), 7.62-7.81(\mathrm{~m}, 2 \mathrm{H}, \mathrm{Ar}) .{ }^{13} \mathrm{C}$ NMR (MeOD, $\left.151 \mathrm{MHz}\right): \delta_{\mathrm{c}}$ 16.51 and 16.72 (each: d, $J_{\mathrm{CP}} 5.3 \mathrm{~Hz}, \mathrm{OCH}_{2} \mathrm{CH}_{3}$ ), 51.85 (d, J $\mathrm{JP}_{\mathrm{CP}} 18.1 \mathrm{~Hz}, \mathrm{CH}_{2} \mathrm{Ph}$ ), 60.01 (d, $J_{\mathrm{CP}} 155.5 \mathrm{~Hz}, \mathrm{CHP}$ ), 64.28 
(d, $J_{\mathrm{CP}} 6.0 \mathrm{~Hz}, \mathrm{OCH}_{2} \mathrm{CH}_{3}$ ), 64.48 (d, $J_{\mathrm{CP}} 7.6 \mathrm{~Hz}, \mathrm{OCH}_{2} \mathrm{CH}_{3}$ ), 128.23 (s, Ar), 128.82 (s, Ar), 129.48 (s, Ar), 129.56 (s, $\operatorname{Ar}), 131.77(\mathrm{~s}, \mathrm{Ar}), 134.72(\mathrm{~s}, \mathrm{Ar}), 135.49$ (s, Ar), 140.39 (s, Ar). Upon recording of MS in methanol this compound forms monomethyl ester (confirmed independently by ${ }^{1} \mathrm{H} N M R$ ), HRMS (ESI-TOF) $m / z$ [MOMe+H ${ }^{+}$, Calcd. for $\mathrm{C}_{19} \mathrm{H}_{28} \mathrm{NO}_{5} \mathrm{BP}$ : 392.1798, found 392.1791.

General procedure for Petasis reaction. To a suspension of diethyl $\alpha$ - $(N-$ benzylamino)boronobenzylphosphonate $(0.6 \mathrm{~g}, 1.6 \mathrm{mmol})$ in appropriate solvent $(12-15 \mathrm{~mL})$ salicylic aldehyde $(0.22 \mathrm{~mL}, 2 \mathrm{mmol})$ or glyoxalic acid $(0.17 \mathrm{mg}, 2 \mathrm{mmol})$ and appropriate amine $(2 \mathrm{mmol})$ were added. Then the mixture was stirred at $80{ }^{\circ} \mathrm{C}$ for $3.5-10 \mathrm{~h}$ (see Results and Discussion) under nitrogen. Volatile components of the reaction mixture were then evaporated and the resulting oil was purified by flash chromatography.

Diethyl $\mathbf{N}$-(benzylamino)-4-[morpholin-1-yl-(2-hydroxyphenyl)methyl]benzylphosphonate (3a) was obtained as a white solidifying oil after flash chromatography using gradient of hexane $/ \mathrm{CHCl}_{3}(0-50 \%)$ as eluent, ${ }^{31} \mathrm{P}$ $\operatorname{NMR}\left(\mathrm{CHCl}_{3}, 121 \mathrm{MHz}\right): \delta_{\mathrm{P}} 23.86$ and 23.90. ${ }^{1} \mathrm{H} \mathrm{NMR}\left(\mathrm{CDCl}_{3}, 600 \mathrm{MHz}\right): \delta_{\mathrm{H}} 1.06-1.10$ and 1.24-1.27 (each: $\mathrm{m}$, $3 \mathrm{H}, \mathrm{OCH}_{2} \mathrm{CH}_{3}$ ), 2.31, 2.46 and 2.62 (each: bs, 4H, $\mathrm{CH}_{2} \mathrm{NHCH}_{2}$ ), 3.54 (d, $\left.J_{\mathrm{HH}} 13.5 \mathrm{~Hz}, 1 \mathrm{H}, \mathrm{NHCH}_{2}\right), 3.66-3.86(\mathrm{~m}$, $5 \mathrm{H}, \mathrm{OCH}_{2} \mathrm{CH}_{3}$ and $\left.\left.\mathrm{CH}_{2} \mathrm{OCH}_{2}\right), 3.79 \mathrm{~d}, J_{\mathrm{HH}} 3.5 \mathrm{~Hz}, 1 \mathrm{H}, \mathrm{NHCH}_{2}\right), 3.90-3.98\left(\mathrm{~m}, 1 \mathrm{H}, \mathrm{OCH}_{2} \mathrm{CH}_{3}\right), 4.01\left(\mathrm{~d}, J_{\mathrm{PH}} 20.7 \mathrm{~Hz}\right.$, $1 \mathrm{H}, \mathrm{CHP}), 4.03-4.11\left(\mathrm{~m}, 2 \mathrm{H}, \mathrm{OCH}_{2} \mathrm{CH}_{3}\right), 4.45(\mathrm{~s}, 1 \mathrm{H}, \mathrm{CH}), 6.74(\mathrm{~m}, 1 \mathrm{H}, \mathrm{Ar}), 6.88$ (ddd, $J_{\mathrm{HH}} 12.1 \mathrm{~Hz}, J_{\mathrm{HH}} 2.9 \mathrm{~Hz}, J_{\mathrm{HH}}$ $1.0 \mathrm{~Hz}, 1 \mathrm{H}, \mathrm{Ar}), 6.97\left(\mathrm{~d}, \mathrm{~J}_{\mathrm{HH}} 6.8 \mathrm{~Hz}, 1 \mathrm{H}, \mathrm{Ar}\right), 7.12-7.16(\mathrm{~m}, 1 \mathrm{H}, \mathrm{Ar}), 7.23-7.26(\mathrm{~m}, 3 \mathrm{H}, \mathrm{Ar}), 7.28-7.31(\mathrm{~m}, 2 \mathrm{H}, \mathrm{Ar})$, 7.39-7.40 (m, 2H, Ar), 7.44-7.45 (m, 2H, Ar). ${ }^{13} \mathrm{C} \mathrm{NMR}\left(\mathrm{CDCl}_{3}, 151 \mathrm{MHz}\right): \delta_{\mathrm{C}} 16,18\left(\mathrm{t}, \mathrm{J}_{\mathrm{CP}} 6.0 \mathrm{~Hz}, \mathrm{OCH}_{2} \mathrm{CH}_{3}\right), 16.42$ (dd, $J_{\mathrm{CP}} 5.3 \mathrm{~Hz}, J_{\mathrm{CP}} 2.2 \mathrm{~Hz}, \mathrm{OCH}_{2} \mathrm{CH}_{3}$ ), 51.43 (d, $J_{\mathrm{CP}} 16.6 \mathrm{~Hz}, \mathrm{NHCH}_{2}$ ), 52.23 (bs, $\mathrm{CH}_{2} \mathrm{OCH}_{2}$ ),

$59.36\left(\mathrm{~d}, J_{\mathrm{CP}} 153.3 \mathrm{~Hz}, \mathrm{CHP}\right), 62.71$ (dd, $J_{\mathrm{CP}} 6.8 \mathrm{~Hz}, J_{\mathrm{CP}} 3.7 \mathrm{~Hz}, \mathrm{OCH}_{2} \mathrm{CH}_{3}$ ), 63.02 (d, J $\left.J_{\mathrm{C}-\mathrm{P}} 8.1 \mathrm{~Hz}, \mathrm{OCH}_{2} \mathrm{CH}_{3}\right), 66.88$

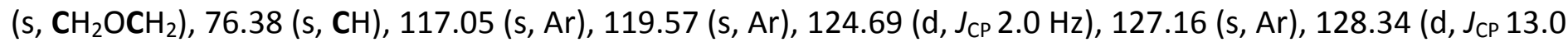
$\mathrm{Hz}, \mathrm{Ar}$ ), 128.73 (bs, Ar), 129.18 (d, $J_{\mathrm{CP}} 5.8 \mathrm{~Hz}, \mathrm{Ar}$ ), 129.44 (s, Ar), 135.86 (d, $J_{\mathrm{C}-\mathrm{p}} 2.6 \mathrm{~Hz}, \mathrm{Ar}$ ), 138.85 (d, Jc-p $2.6 \mathrm{~Hz}$, $\operatorname{Ar}), 139.28(\mathrm{~s}, \mathrm{Ar}), 156.14(\mathrm{~s}, \mathrm{Ar})$. HRMS (ESI-TOF) m/z [M+H $\mathrm{H}^{+}$, Calcd. for $\mathrm{C}_{29} \mathrm{H}_{38} \mathrm{~N}_{2} \mathrm{O}_{5} \mathrm{P}$ : 525.2518, found 525.2523.

Diethyl $\mathbf{N}$-(benzylamino)-4-[piperidin-1-yl-(2-hydroxyphenyl)methyl]benzylphosphonate (3b) was obtained after purification by flash chromatography using mixture of $\mathrm{CHCl}_{3} / \mathrm{MeOH}(15: 1)$ as dense yellow oil, ${ }^{31} \mathrm{P} N M R$ (MeOD, $243 \mathrm{MHz}$ ): $\delta_{\mathrm{P}} 23.77$ (bs). ${ }^{1} \mathrm{H}$ NMR (MeOD, $600 \mathrm{MHz}$ ): $\delta_{\mathrm{H}} 1.06$ and 1.07 (each: t, $J_{\mathrm{HH}} 7.3 \mathrm{~Hz}, 1.5 \mathrm{H}$, $\mathrm{OCH}_{2} \mathrm{CH}_{3}$ ), 1.26 (t, JH $7.0 \mathrm{~Hz}, 3, \mathrm{OCH}_{2} \mathrm{CH}_{3}$ ), 1.50 (bs, 2H, NCH $\mathrm{CH}_{2} \mathrm{CH}_{2}$ ), 1.64 (bs, 4H, $\mathrm{NCH}_{2} \mathrm{CH}_{2} \mathrm{CH}_{2}$ ), 2.35-2.64 (m, $4 \mathrm{H}, \mathrm{NCH}_{2} \mathrm{CH}_{2} \mathrm{CH}_{2}$ ), 3.70 ( $\mathrm{AB}$ system, $\mathrm{J}_{\mathrm{HH}} 12.1 \mathrm{~Hz}, \mathrm{~J}_{\mathrm{HH}} 156.3 \mathrm{~Hz}, 2 \mathrm{H}, \mathrm{CH}_{2} \mathrm{Ph}$ ), 3.73-3.81 and 3.85-3.94 (each: $\mathrm{m}$, $1 \mathrm{H}, \mathrm{OCH}_{2} \mathrm{CH}_{3}$ ), 4.02 (d, $J_{\mathrm{PH}} 18.2 \mathrm{~Hz}, 1 \mathrm{H}, \mathrm{CHP}$ ), 4.07 and 4.08 (each: pentet, $\left.1 \mathrm{H}, J_{\mathrm{CP}}=J_{\mathrm{HH}} 6.8 \mathrm{~Hz}, \mathrm{OCH}_{2} \mathrm{CH}_{3}\right), 4.57(\mathrm{~s}$, $1 \mathrm{H}, \mathrm{CH}), 6.70\left(\mathrm{t}, J_{\mathrm{HH}} 6.0 \mathrm{~Hz}, 1 \mathrm{H}, \mathrm{Ar}\right), 6.80\left(\mathrm{~d}, J_{\mathrm{HH}} 6.0 \mathrm{~Hz}, 1 \mathrm{H}, \mathrm{Ar}\right), 6.99\left(\mathrm{~d}, J_{\mathrm{HH}} 6.0 \mathrm{~Hz}, 1 \mathrm{H}, \mathrm{Ar}\right), 7.08\left(\mathrm{t}, J_{\mathrm{HH}} 6.0 \mathrm{~Hz}\right.$, $1 \mathrm{H}, \mathrm{Ar}$ ), $7.23\left(\mathrm{~d}, J_{\mathrm{HH}} 6.0 \mathrm{~Hz}, 2 \mathrm{H} \mathrm{Ar}\right), 7.28\left(\mathrm{~d}, J_{\mathrm{HH}} 6.0 \mathrm{~Hz}, 2 \mathrm{H} \mathrm{Ar}\right), 7.38\left(\mathrm{~d}, J_{\mathrm{HH}} 6.0 \mathrm{~Hz}, 2 \mathrm{H} \mathrm{Ar}\right), 7.48\left(\mathrm{~d}, J_{\mathrm{HH}} 6.0 \mathrm{~Hz}, 2 \mathrm{H}\right.$ Ar). HRMS (ESI-TOF) m/z [M+H'] , Calcd. for $\mathrm{C}_{30} \mathrm{H}_{40} \mathrm{~N}_{2} \mathrm{O}_{4} \mathrm{P}: 523.2726$, found 523.2711.

Diethyl $\mathbf{N}$-(benzylamino)-4-[bis(dimethoxyethyl)(2-hydroxyphenyl)methyl]benzylphosphonate (3c) was obtained after purification by flash chromatography using mixture of $\mathrm{CHCl}_{3} / \mathrm{MeOH}(15: 1)$ as dense yellow oil, ${ }^{31} \mathrm{P}$ NMR (MeOD, $243 \mathrm{MHz}$ ): $\delta_{\mathrm{P}} 23.77$ and 23.95. ${ }^{1} \mathrm{H}$ NMR (MeOD, $600 \mathrm{MHz}$ ): $\delta_{\mathrm{H}} 1.10$ and 1.11 (each: t, $J_{\mathrm{HH}} 7.0$ $\mathrm{Hz}, 1.5 \mathrm{H}, \mathrm{OCH}_{2} \mathrm{CH}_{3}$ ), $1.29\left(\mathrm{t}, \mathrm{J}_{\mathrm{HH}} 7.0 \mathrm{~Hz}, 3, \mathrm{OCH}_{2} \mathrm{CH}_{3}\right.$ ), 2.79-2.81 and 2.94-2.97 (each: $\mathrm{m}, 2 \mathrm{H}, \mathrm{NCH}_{2} \mathrm{CH}_{2}$ ), 3.32 and 3.33 (each: $\left.\mathrm{s}, 3 \mathrm{H}, \mathrm{OCH}_{3}\right), 3.42(\mathrm{~s}, \mathrm{CHCOOH}), 3.47-3.49\left(\mathrm{~m}, 2 \mathrm{H}, \mathrm{OCH}_{2}\right), 3.53-3.55\left(\mathrm{~m}, 3 \mathrm{H}, \mathrm{POCH}_{2}\right.$ and $\left.\mathrm{NCH}_{2}\right), 3.81$ (t, $J_{\mathrm{HH}} 13.7 \mathrm{~Hz} 3 \mathrm{H}, \mathrm{CH}_{2} \mathrm{Ph}$ ), 3.88-4.00 and 4.04-4.14 (each: $\mathrm{m}, 2 \mathrm{H}, \mathrm{POCH}_{2}$ ), 4.06, (d, $\left.\mathrm{JPH}_{\mathrm{PH}} 22.6 \mathrm{~Hz}, 1 \mathrm{H}, \mathrm{CHP}\right), 6.74(\mathrm{t}$, $\left.J_{H H} 7.5 \mathrm{~Hz}, 1 \mathrm{H}, \mathrm{Ar}\right), 6.81\left(\mathrm{~d}, J_{\mathrm{HH}} 8.1 \mathrm{~Hz}, 1 \mathrm{H}, \mathrm{Ar}\right), 6.90\left(\mathrm{~d}, J_{\mathrm{HH}} 7.6 \mathrm{~Hz}, 1 \mathrm{H}, \mathrm{Ar}\right), 7.81\left(\mathrm{t}, J_{\mathrm{HH}} 7.7 \mathrm{~Hz}, 1 \mathrm{H}, \mathrm{Ar}\right), 7.27\left(\mathrm{~d}, J_{\mathrm{HH}}\right.$ $\left.6.9 \mathrm{~Hz}, 4 \mathrm{H}, \mathrm{Ar}), 7.31\left(\mathrm{~s}, J_{\mathrm{HH}} 7.9 \mathrm{~Hz}, 2 \mathrm{H}, \mathrm{Ar}\right), 7.44\left(\mathrm{~s}, J_{\mathrm{HH}} 7.2 \mathrm{~Hz}, 2 \mathrm{H}, \mathrm{Ar}\right), 7.51\left(\mathrm{~s}, J_{\mathrm{HH}} 7.8 \mathrm{~Hz}, 1 \mathrm{H}, \mathrm{Ar}\right).\right) .{ }^{13} \mathrm{C} \mathrm{NMR}$ ( $\mathrm{CDCl}_{3}, 151 \mathrm{MHz}$ ): $\delta_{\mathrm{C}} 16,18$ (t, $J_{\mathrm{CP}} 15.2 \mathrm{~Hz}, \mathrm{OCH}_{2} \mathrm{CH}_{3}$ ), 29.68 (s, $\mathrm{CH}_{2} \mathrm{Ph}$ ), 50.19 (bs, OCH3), 50.75 and 50.77 (each: s, $\mathrm{CH}_{2} \mathrm{OCH}_{3}$ ), 57.60 (s, $\mathrm{CHCOOH}$ ), 58.52 and 58.53 (each: d, $J_{\mathrm{CP}} 155.4 \mathrm{~Hz}, \mathrm{CHP}$ ), 63.03 and 63.09 (each d, J $J_{\mathrm{CP}} 25.6$ $\mathrm{Hz}, \mathrm{POCH}_{2}$ ), 69.74 (s, $\mathrm{CHCOOH}$ ), 70.04 (s, $\mathrm{NHCH}_{2}$ ), 116.13 (s, Ar), 118.94 (s, Ar), 126.87 (s, Ar), 128.04 (s, Ar), 
128.21 (s, Ar), 128.25 (s, Ar), 128.83 (d, J JP $6.2 \mathrm{~Hz}$ ), 128.96 (s, Ar), 129.50 (d, J JP $1.5 \mathrm{~Hz}$ ), 139.09 (s, Ar), 156.56 (s, Ar), 203.23 (s, COOH). HRMS (ESI-TOF) m/z [M+H ${ }^{+}$, Calcd. for $\mathrm{C}_{31} \mathrm{H}_{44} \mathrm{~N}_{2} \mathrm{O}_{6} \mathrm{P}:$ 571.2937, found 571.2142.

Diethyl $\mathbf{N}$-(benzylamino)-3-[morpholin-1-yl-(2-hydroxyphenyl)methyl]benzylphosphonate (4a) was obtained as a dense yellowish oil after flash chromatography using mixure of hexane/EtOAc (3:7) as eluent, ${ }^{31} \mathrm{P} N M R$ $\left(\mathrm{CHCl}_{3}, 121 \mathrm{MHz}\right): 21.84$ and $21.97{ }^{1} \mathrm{H} \mathrm{NMR}\left(\mathrm{CDCl}_{3}, 600 \mathrm{MHz}\right): \delta_{\mathrm{H}} 1.16-1.19\left(\mathrm{~m}, 3 \mathrm{H}, \mathrm{OCH}_{2} \mathrm{CH}_{3}\right), 1,32\left(\mathrm{t}, J_{\mathrm{HH}} 7.0\right.$ $\mathrm{Hz}, 3 \mathrm{H}, \mathrm{OCH}_{2} \mathrm{CH}_{3}$ ), 2.78 and 3.07 (each: bs, $1 \mathrm{H}, \mathrm{CH}_{2}$ ), 2.36 (bs, $2 \mathrm{H}, \mathrm{NCH}_{2}$ ), 3.68 (AB system, $J_{\mathrm{HH}} 13.5 \mathrm{~Hz}, J_{\mathrm{HH}}$ $\left.158.6 \mathrm{~Hz}, 2 \mathrm{H}, \mathrm{CH}_{2} \mathrm{Ph}\right), 3.66-3.86\left(\mathrm{~m}, 5 \mathrm{H}, \mathrm{POCH}_{2}\right.$ and $\left.\mathrm{CH}_{2} \mathrm{OCH}_{2}\right), 3.90-3.38\left(\mathrm{~m}, \mathrm{POCH}_{2}\right), 4.01\left(\mathrm{~d}, J_{\mathrm{PH}} 20.7 \mathrm{~Hz}, 1 \mathrm{H}\right.$, CHP), 4.03-4.11 (m, 2H, POCH $), 4.45(\mathrm{~s}, 1 \mathrm{H}, \mathrm{CH}), 6.74\left(\mathrm{t}, J_{\mathrm{HH}} 6.0 \mathrm{~Hz}, \mathrm{Ar}\right), 6.88$ (ddd, $J_{\mathrm{HH}} 12.1 \mathrm{~Hz}, J_{\mathrm{HH}} 2.9 \mathrm{~Hz}, J_{\mathrm{HH}} 1.0$ $\mathrm{Hz}, 1 \mathrm{H}, \mathrm{Ar}), 6.97$ (d, $\left.J_{\mathrm{HH}} 6.8 \mathrm{~Hz}, \mathrm{Ar}\right), 7.12-7.16(\mathrm{~m}, 1 \mathrm{H} \mathrm{Ar}), 7.23-7.26$ (m, 3H Ar), 7.28-7.31 (m, 2H Ar), 7.39-7.40

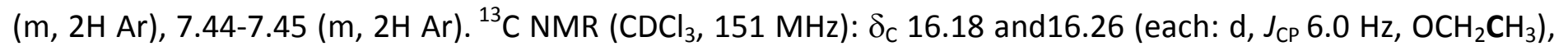
16.40 and 16.44 (each :d, $J_{\mathrm{CP}} 5.3 \mathrm{~Hz}, \mathrm{OCH}_{2} \mathrm{CH}_{3}$ ), 51.36 and 51.42 (each : d, $\mathrm{CH}_{2} \mathrm{Ph}$ ), $52.23\left(\mathrm{bs}, \mathrm{CH}_{2} \mathrm{OCH}_{2}\right.$ ), 59.36 (d, $J_{\mathrm{CP}} 153.3 \mathrm{~Hz}, \mathrm{CHP}$ ), 62.70 and 62.74 (each: d, $\left.J_{\mathrm{CP}} 6.8 \mathrm{~Hz}, \mathrm{OCH}_{2} \mathrm{CH}_{3}\right), 63.02$ (d, $\left.J_{\mathrm{CP}} 8.1 \mathrm{~Hz}, \mathrm{OCH}_{2} \mathrm{CH}_{3}\right), 66.88(\mathrm{~s}$,

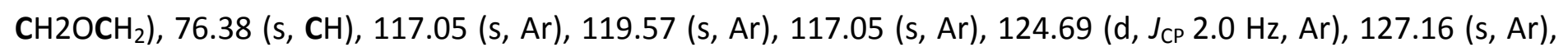
128.34 (d, J JP $13.0 \mathrm{~Hz}, \mathrm{Ar}$ ), 128.73 (bs, Ar), 129.18 (d, $J_{\mathrm{CP}} 13.0 \mathrm{~Hz}, \mathrm{Ar}$ ), 129.44 (bs, Ar), 135.86 (d, J $J_{\mathrm{CP}} .6 \mathrm{~Hz}, \mathrm{Ar}$ ), 138.85 (d, $J_{C P} 2.6 \mathrm{~Hz}, \mathrm{Ar}$ ), 139.28 (s, Ar), 156.14 (s, Ar). HRMS (ESI-TOF) m/z [M+H'], Calcd. for $\mathrm{C}_{29} \mathrm{H}_{38} \mathrm{~N}_{2} \mathrm{O}_{5} \mathrm{P}$ : 525.2518 , found 525.2519 .

Diethyl $\mathbf{N}$-(benzylamino)-3-[piperidin-1-yl-(2-hydroxyphenyl)methyl]benzylphosphonate (4b) was obtained as a dense yellowish oil after flash chromatography using mixture of $\mathrm{CHCl}_{3} / \mathrm{MeOH}(15: 1)$ as eluent, ${ }^{31} \mathrm{P} \mathrm{NMR}$ $\left(\mathrm{CHCl}_{3}, 121 \mathrm{MHz}\right): \delta_{\mathrm{p}} 23,20$ and 23,26 (diastereoizomers) and additional rotamers at 23,51. ${ }^{1} \mathrm{H} \mathrm{NMR}\left(\mathrm{CDCl}_{3}, 600\right.$ $\mathrm{MHz}$ ): $\delta_{\mathrm{H}} 1.07$ and 1.12 (each: $t, 3 \mathrm{H}, \mathrm{OCH}_{2} \mathrm{CH}_{3}$ ), 1.16 and 1.30 (each: $\left.t, 3 \mathrm{H}, \mathrm{OCH}_{2} \mathrm{CH}_{3}\right), 1.25-1.28(\mathrm{~m}, 6 \mathrm{H}$, $\mathrm{OCH}_{2} \mathrm{CH}_{3}$ ), 1.49 (bs, $2 \mathrm{H}, \mathrm{NCH}_{2} \mathrm{CH}_{2} \mathrm{CH}_{2}$ ), 1.68 (bs, 8H, $\mathrm{CH}_{2} \mathrm{CH}_{2} \mathrm{NCH}_{2} \mathrm{CH}_{2}$ ), $3.50-3.54$ (m, 2H, NHCH ), 3.57 (d, J JH $\left.18.0 \mathrm{~Hz}, 1 \mathrm{H}, \mathrm{NHCH}_{2}\right), 3.72-4.15\left(\mathrm{~m}, 16 \mathrm{H}, \mathrm{OCH}_{2} \mathrm{CH}_{3}+\mathrm{CHP}\right), 6.71\left(\mathrm{q}, 2 \mathrm{H}, J_{\mathrm{HH}} 6.0 \mathrm{~Hz}, \mathrm{Ar}\right), 6.86-6.91(\mathrm{~m}, 2 \mathrm{H}, \mathrm{Ar})$, 6.95 (bs, 2H, Ar), $7.10-7.15(\mathrm{~m}, 2 \mathrm{H}, \mathrm{Ar}), 7.21-7.24(\mathrm{~m}, 4 \mathrm{H}, \mathrm{Ar}), 7.27-7.42(\mathrm{~m}, 17 \mathrm{H}, \mathrm{Ar}), 7.45-7.48(\mathrm{~m}, 3 \mathrm{H}$, $\mathrm{Ar}), 12.47$ (bs, $1 \mathrm{H}, \mathrm{OH}) .{ }^{13} \mathrm{C} \mathrm{NMR}\left(\mathrm{CDCl}_{3}, 151 \mathrm{MHz}\right): \delta_{\mathrm{C}} 16.25$ (d, J $\left.J_{\mathrm{CP}} 6.0 \mathrm{~Hz}, \mathrm{OCH}_{2} \mathrm{CH}_{3}\right), 16.33\left(\mathrm{~d}, J_{\mathrm{CP}} 6.0 \mathrm{~Hz}\right.$, $\mathrm{OCH}_{2} \mathrm{CH}_{3}$ ), 16.47 (d, $J_{\mathrm{CP}} 4.5 \mathrm{~Hz}, \mathrm{OCH}_{2} \mathrm{CH}_{3}$, rotamer), 51.24 (d, $J_{\mathrm{CP}} 24.2 \mathrm{~Hz}, \mathrm{NHCH}_{2}$ ), 51.35 (d, J $J_{\mathrm{CP}} 24.2 \mathrm{~Hz}, \mathrm{NHCH}_{2}$ ), 52.20 (bs, $\mathrm{CH}_{2} \mathrm{NCH}_{2}$ ), 59.39 (d, $J_{\mathrm{CP}} 152.5 \mathrm{~Hz}, \mathrm{CHP}$ ), 59.64 (d, $J_{\mathrm{CP}} 151.0 \mathrm{~Hz}, \mathrm{CHP}$ ), 62.72 (d, J $\mathrm{JP}_{\mathrm{CP}} 6.0 \mathrm{~Hz}, \mathrm{OCH}_{2} \mathrm{CH}_{3}$ ), 62.73-63.00 (m, OCH $\left.\mathrm{CH}_{3}\right), 66.86\left(\mathrm{~s}, \mathrm{CH}_{2} \mathrm{OCH}_{2}\right), 66.89\left(\mathrm{~s}, \mathrm{CH}_{2} \mathrm{OCH}_{2}\right), 76.57(\mathrm{~s}, \mathrm{CH}), 76.60(\mathrm{~s}, \mathrm{CH}), 117.07$ (s, Ar), 119.57(s, Ar), 119.58 (s, Ar), 124.67 (s, Ar), 127.23 (s, Ar), 127.96 (bs, Ar), 128.38-128.43 (m, Ar), 128.73 (s, Ar), 129.18 (bs, Ar), 129.33 (d, J JP $6.0 \mathrm{~Hz}, \mathrm{Ar}$ ), 136.56 (bs, Ar), 139.08 (s, Ar), 139.13 (s, Ar), 139.47 (d, J $J_{\mathrm{CP}} 9.0 \mathrm{~Hz}, \mathrm{Ar}$ ), 156.10 (s, Ar), 156.13 (s, Ar). HRMS (ESI-TOF) m/z [M+H'] , Calcd. for $\mathrm{C}_{30} \mathrm{H}_{40} \mathrm{~N}_{2} \mathrm{O}_{4} \mathrm{P}:$ 523.2726, found 523.2699.

Diethyl ( $\mathbf{N}$-benzylamino)-4-morpholin-1-yl-(carboxymethyl)benzylphosphonate (5a) was obtained as a

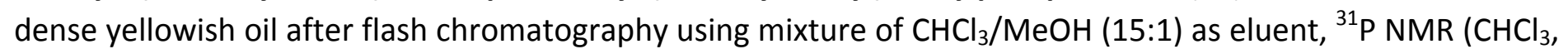
$121 \mathrm{MHz}$ ): $\delta_{\mathrm{P}} 23.60 .{ }^{1} \mathrm{H}$ NMR (MeOD, $600 \mathrm{MHz}$ ): $\delta_{\mathrm{H}}$ 1.09-1.12 and 1.23-1.26 (each: $\mathrm{m}, 3 \mathrm{H}, \mathrm{OCH}_{2} \mathrm{CH}_{3}$ ), 2.92 and 2.90 (each: s, $2 \mathrm{H}, \mathrm{CH}_{2} \mathrm{NCH}_{2}$ ), 3.51 (d, $\left.\mathrm{J}_{\mathrm{HH}} 13.7 \mathrm{~Hz}, 1 \mathrm{H}, \mathrm{CH}_{2} \mathrm{Ph}\right), 3.71-3.80\left(\mathrm{~m}, 5 \mathrm{H}, \mathrm{CH}_{2} \mathrm{Ph}, \mathrm{CH}_{2} \mathrm{OCH}_{2}\right), 3.81-3.86$ and 3.91-3.97 (each: $\left.\mathrm{m}, 1 \mathrm{H}, \mathrm{OCH}_{2} \mathrm{CH}_{3}\right), 4.02$ (d, $\left.\mathrm{JPH}_{\mathrm{PH}} 21.2 \mathrm{~Hz}, 1 \mathrm{H}, \mathrm{CHP}\right), 4.02-4.10\left(\mathrm{~m}, 2 \mathrm{H}, \mathrm{OCH}_{2} \mathrm{CH}_{3}\right), 4.43$. (s, 1H, $\mathrm{CH}), 5.71(\mathrm{bs}, 2 \mathrm{H}, \mathrm{NH}$ and $\mathrm{OH}), 7.21-7.23(\mathrm{~m}, 3 \mathrm{H}, \mathrm{Ar}), 7.26-7.29(\mathrm{~m}, 2 \mathrm{H}, \mathrm{Ar}), 7.43\left(\mathrm{~d}, \mathrm{~J}_{\mathrm{HH}} 7.7 \mathrm{~Hz}, 2 \mathrm{H}, \mathrm{Ar}\right), 7.53(\mathrm{~d}$, $\left.J_{\mathrm{HH}} 7.9 \mathrm{~Hz}, 2 \mathrm{H}, \mathrm{Ar}\right) .{ }^{13} \mathrm{C} \mathrm{NMR}\left(\mathrm{CDCl}_{3}, 151 \mathrm{MHz}\right): \delta_{\mathrm{C}} 16.25\left(\mathrm{~d}, J_{\mathrm{CP}} 5.4 \mathrm{~Hz}, \mathrm{OCH}_{2} \mathrm{CH}_{3}\right), 16.43\left(\mathrm{~d}, J_{\mathrm{CP}} 5.7 \mathrm{~Hz}_{,} \mathrm{OCH}_{2} \mathrm{CH}_{3}\right)$, 43.6 (s, $\mathrm{CH}_{2}$ ), 51.24-51.35 (m, $\mathrm{CH}_{2} \mathrm{Ph}$ ), 59.11 (d, $\left.J_{\mathrm{CP}} 153.4 \mathrm{~Hz}, \mathrm{CHP}\right), 6297$ (d, J $\left.J_{\mathrm{CP}} 6.7 \mathrm{~Hz}, \mathrm{OCH}_{2} \mathrm{CH}_{3}\right), 6228$ (d, J $J_{\mathrm{CP}} 7.3$ $\mathrm{Hz}, \mathrm{OCH}_{2} \mathrm{CH}_{3}$ ), 64.96 (s, $\mathrm{CH}_{2}$ ), 74.61, (s, CHCOOH), 127.22 (s, Ar), 128.30 (s, Ar), 128.41 (s, Ar), 129.14 (d, J JP 4.7 $\mathrm{Hz}, \mathrm{Ar}$ ), 129.75 (s, Ar), 132.78 (s, Ar), 136.85 (s, Ar), 139.06 (s, Ar), 171.20 (s, COOH), HRMS (ESI-TOF) m/z $\left[\mathrm{M}+\mathrm{H}^{+}\right]$, Calcd. for $\mathrm{C}_{24} \mathrm{H}_{34} \mathrm{~N}_{2} \mathrm{O}_{6} \mathrm{P}: 477.2155$, found 477.2133.

Diethyl ( $\mathbf{N}$-benzylamino)-4-piperidin-1-yl-(carboxymethyl)benzylphosphonate (5b) was obtained as a dense lightly yellow oil after flash chromatography using mixture of $\mathrm{CHCl}_{3} / \mathrm{MeOH}(15: 1)$ as eluent, ${ }^{31} \mathrm{P} \mathrm{NMR}\left(\mathrm{CHCl}_{3}\right.$, $121 \mathrm{MHz}$ ): $\delta_{\mathrm{P}} 23.31$ and 23.32. ${ }^{1} \mathrm{H}$ NMR (MeOD, $600 \mathrm{MHz}$ ): $\delta_{\mathrm{H}} 1.168$ and 1.172 (each: $\mathrm{t}, J_{\mathrm{HH}} 7.0 \mathrm{~Hz}, 1.5 \mathrm{H}$, 
$\mathrm{OCH}_{2} \mathrm{CH}_{3}$ ), 1.31 (t, $J_{\mathrm{HH}} 7.0 \mathrm{~Hz}, 3 \mathrm{H}, \mathrm{OCH}_{2} \mathrm{CH}_{3}$ ), 1.66 (bs, $2 \mathrm{H}, \mathrm{CH}_{2} \mathrm{CH}_{2} \mathrm{CH}_{2}$ ), 1.4-2.1 (m, 7H, NCH $\mathrm{CH}_{2} \mathrm{CH}_{2}$ ), 2.4-3.1 ( $\mathrm{m}, 3 \mathrm{H}, \mathrm{NCH}_{2} \mathrm{CH}_{2} \mathrm{CH}_{2}$ ), 3.68 ( $\mathrm{AB}$ system, $J_{\mathrm{HH}} 13.4 \mathrm{~Hz}, J_{\mathrm{HH}} 159.1 \mathrm{~Hz}, 2 \mathrm{H}, \mathrm{CH}_{2} \mathrm{Ph}$ ), 3.85-3.96 and 3.96-4.00 (each: $\mathrm{m}$, $\left.1 \mathrm{H}, \mathrm{OCH}_{2} \mathrm{CH}_{3}\right), 4.04-4.23\left(\mathrm{~m}, 2 \mathrm{H}, \mathrm{OCH}_{2} \mathrm{CH}_{3}\right), 4.06\left(\mathrm{~d}, J_{\mathrm{PH}} 22.0 \mathrm{~Hz}, 1 \mathrm{H}, \mathrm{CHP}\right), 4.55$ (s, CHCOOH$), 7.26\left(\mathrm{~d}, \mathrm{~J}_{\mathrm{HH}} 7.4 \mathrm{~Hz}\right.$, $2 \mathrm{H}, \mathrm{Ar}$ ), 7.31 (d, $J_{\mathrm{HH}} 7.1 \mathrm{~Hz}, 2 \mathrm{H}, \mathrm{Ar}$ ), 7.41 (s, $1 \mathrm{H}, \mathrm{Ar}$ ), 7.52 (d, $J_{\mathrm{HH}} 7.4 \mathrm{~Hz}, 2 \mathrm{H}, \mathrm{Ar}$ ), 7.63 (d, $J_{\mathrm{HH}} 7.1 \mathrm{~Hz}, 2 \mathrm{H}, \mathrm{Ar}$ ). HRMS (ESI-TOF) $\mathrm{m} / \mathrm{z}\left[\mathrm{M}+\mathrm{H}^{+}\right]$, Calcd. for $\mathrm{C}_{24} \mathrm{H}_{34} \mathrm{~N}_{2} \mathrm{O}_{6} \mathrm{P}: 475.2362$, found 475.2367.

Diethyl ( $\mathrm{N}$-benzylamino)-4-[bis(dimethoxyethyl)(carboxymethyl)]benzylphosphonate (5c) was obtained as a dense yellow oil after flash chromatography using mixture of $\mathrm{CHCl}_{3} / \mathrm{MeOH}(15: 1)$ as eluent, ${ }^{31} \mathrm{P} \mathrm{NMR}$ (MeOD, $243 \mathrm{MHz}$ ): $\delta_{\mathrm{P}} 23.35$ and 23.36. ${ }^{1} \mathrm{H}$ NMR (MeOD, $600 \mathrm{MHz}$ ): $\delta_{\mathrm{H}} 1.17$ and 1.31 (each: t, $J_{\mathrm{HH}} 7.0 \mathrm{~Hz}, 3 \mathrm{H}, \mathrm{OCH}_{2} \mathrm{CH}_{3}$ ), 3.17-3.21 and 3.40-3.46 (each: $\left.\mathrm{m}, 2 \mathrm{H}, \mathrm{NCH}_{2} \mathrm{CH}_{2}\right), 3.33\left(\mathrm{~s}, 6 \mathrm{H}, \mathrm{OCH}_{3}\right), 3.42(\mathrm{~s}, 1 \mathrm{H}, \mathrm{CHCOOH}), 3.60(\mathrm{AB}$ system, JH $20.1 \mathrm{~Hz}, \mathrm{~J}_{\mathrm{HH}} 148.2 \mathrm{~Hz}, 2 \mathrm{H}, \mathrm{CH}_{2} \mathrm{Ph}$ ), 3.69-3.66 and 3.66-3.74 (each: $\mathrm{m}, 2 \mathrm{H}, \mathrm{NCH}_{2}$ ), 3.85-3.95 and 3,95-4,03 (each : $\left.\mathrm{m}, 1 \mathrm{H}, \mathrm{POCH}_{2}\right), 4.03-4.17\left(\mathrm{~m}, 2 \mathrm{H}, \mathrm{POCH}_{2}\right), 4.10$ (d, $\left.J_{\mathrm{PH}} 23.8 \mathrm{~Hz}, 1 \mathrm{H}, \mathrm{CHP}\right), 7.26$ (bd, $\left.J_{\mathrm{HH}} 6.0 \mathrm{~Hz}, 2 \mathrm{H}, \mathrm{Ar}\right), 7.32(\mathrm{~d}$, $\left.J_{\mathrm{HH}} 6.0 \mathrm{~Hz}, 2 \mathrm{H}, \mathrm{Ar}\right), 7.51\left(\mathrm{~d}, J_{\mathrm{HH}} 6.0 \mathrm{~Hz}, 2 \mathrm{H}, \mathrm{Ar}\right), 7.61\left(\mathrm{~d}, J_{\mathrm{HH}} 6.0 \mathrm{~Hz}, 2 \mathrm{H}, \mathrm{Ar}\right) .{ }^{13} \mathrm{C}$ NMR (MeOD, $\left.151 \mathrm{MHz}\right): \delta_{\mathrm{C}} 15.22$ and 15.35 (each: d, $J_{\mathrm{CP}} 5.8 \mathrm{~Hz}, \mathrm{OCH}_{2} \mathrm{CH}_{3}$ ), 29.28 (s, $\mathrm{CH}_{2} \mathrm{Ph}$ ), 50.70 and 50.72 (each: $\mathrm{s}, \mathrm{CH}_{2} \mathrm{OCH}_{3}$ ), 51.43 (s, $\mathrm{CH}$ ), $57.87(\mathrm{~s}, \mathrm{OCH} 3), 58.50$ (d, $\left.J_{\mathrm{CP}} 154.4 \mathrm{~Hz}, \mathrm{CHP}\right), 60.01$ (d, $\left.J_{\mathrm{CP}} 7.2 \mathrm{~Hz}, \mathrm{OCH}_{2} \mathrm{CH}_{3}\right), 63.22\left(\mathrm{~d}, J_{\mathrm{CP}} 7.0 \mathrm{~Hz}, \mathrm{OCH}_{2} \mathrm{CH}_{3}\right)$, 66.94 (s, CHCOOH), 72.46 (s, $\mathrm{NHCH}_{2}$ ), 126.90 (s, Ar), 128.05 (s, Ar), 128.15 (s, Ar), 128.19 (s, Ar), 128.93 (s, Ar), 128.76 (d, J JP $3.2 \mathrm{~Hz}, \mathrm{Ar}$ ), 129.15 (d, J $J_{\mathrm{CP}} 6.1 \mathrm{~Hz}, \mathrm{Ar}$ ), 130.09 (s, Ar), 130.28 (s, Ar), 137.17 (s, Ar), 139.03 (s, Ar), 208.37 (s, COOH). HRMS (ESI-TOF) m/z [M+H ${ }^{+}$, Calcd. for $\mathrm{C}_{26} \mathrm{H}_{42} \mathrm{~N}_{2} \mathrm{O} 6$ P: 509.2781, found 509.2795.

Diethyl ( $\mathbf{N}$-benzylamino)-3-morpholin-1yl-(carboxymethyl)benzylphosphonate (6a) was obtained as a dense yellowish oil after flash chromatography using mixture of $\mathrm{CHCl}_{3} / \mathrm{MeOH}(15: 1)$ as eluent, ${ }^{31} \mathrm{P} \mathrm{NMR}\left(\mathrm{CHCl}_{3}, 243\right.$ $\mathrm{MHz}): \delta_{\mathrm{P}} 21,84$ and 21,97. ${ }^{1} \mathrm{H} \mathrm{NMR}\left(\mathrm{CDCl}_{3}, 600 \mathrm{MHz}\right): \delta_{\mathrm{H}} 1.16-1.19\left(\mathrm{~m}, 3 \mathrm{H}, \mathrm{OCH}_{2} \mathrm{CH}_{3}\right), 1.32\left(\mathrm{t}, \mathrm{J}_{\mathrm{HH}} 7.0 \mathrm{~Hz}, 3 \mathrm{H}\right.$, $\mathrm{OCH}_{2} \mathrm{CH}_{3}$ ), 2.78 and 3.07 (each: bs, $2 \mathrm{H}, \mathrm{CH}_{2} \mathrm{NHCH}_{2}$ ), 3.56 (bd, $\mathrm{J}_{\mathrm{HH}} 12.0 \mathrm{~Hz}, 1 \mathrm{H}, \mathrm{CH}_{2} \mathrm{Ph}$ ), 3.80-3.91 (m, 6H, CH $\mathrm{Ch}_{2}$ $\mathrm{OCH}_{2} \mathrm{CH}_{3}$ and $\left.\mathrm{CH}_{2} \mathrm{OCH}_{2}\right), 3.95-4.03\left(\mathrm{~m}, 1 \mathrm{H}, \mathrm{OCH}_{2} \mathrm{CH}_{3}\right), 4.07-4,19\left(\mathrm{~m}, 3 \mathrm{H}, \mathrm{CHP}\right.$ and $\left.\mathrm{OCH}_{2} \mathrm{CH}_{3}\right), 4.26$ and 4.28 (each : s, 1H, CHCOOH), 7.26-7.30 (m, 3H, Ar), 7.32-7.36 (m, 2H, Ar), 7.43-7.52 (m, 2H, Ar), $7.56\left(\mathrm{~d}, J_{\mathrm{HH}} 6.0 \mathrm{~Hz}\right.$,

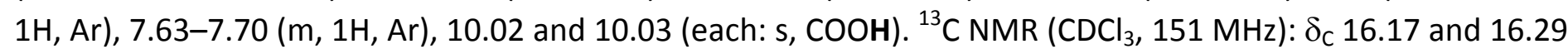
(each: d, $J_{\mathrm{CP}} 6.0 \mathrm{~Hz}, \mathrm{OCH}_{2} \mathrm{CH}_{3}$ ), $16.44\left(\mathrm{t}, J_{\mathrm{CP}} 6.0 \mathrm{~Hz}, \mathrm{OCH}_{2} \mathrm{CH}_{3}\right), 51.24$ (d, $J_{\mathrm{CP}} 15.1 \mathrm{~Hz}, \mathrm{NHCH}_{2}$ ), $51.58\left(\mathrm{bs}, \mathrm{CH}_{2} \mathrm{NCH}_{2}\right.$ ), $59.00\left(\mathrm{~d}, J_{\mathrm{CP}} 152.5 \mathrm{~Hz}, \mathrm{CHP}\right), 59.17\left(\mathrm{~d}, J_{\mathrm{CP}} 154.0 \mathrm{~Hz}, \mathrm{CHP}\right), 62.87$ (d, $\left.J_{\mathrm{C} . \mathrm{P}} 6.0 \mathrm{~Hz}, \mathrm{OCH}_{2} \mathrm{CH}_{3}\right), 63.06\left(\mathrm{~d}, J_{\mathrm{CP}} 6.0 \mathrm{~Hz}\right.$, $\mathrm{OCH}_{2} \mathrm{CH}_{3}$ ), 63.30 (bs, $\mathrm{CH}_{2} \mathrm{OCH}_{2}$ ), 75.99 (bs, CH), 127.21 (d, $J_{\mathrm{CP}} 4.5 \mathrm{~Hz}, \mathrm{Ar}$ ), 128.32 (s, Ar), 128.39 (s, Ar), 128.44 (s, Ar), 128.66 (s, Ar), 128.77 (s, Ar), 136.02 (bs, Ar), 136.35 (s, Ar), 139.18 (d, J JP $9.0 \mathrm{~Hz}, \mathrm{Ar}$ ). Calcd. for $\mathrm{C}_{24} \mathrm{H}_{34} \mathrm{~N}_{2} \mathrm{O}_{6} \mathrm{P}: 477.2155$, found 477.2165. HRMS (ESI-TOF) $\mathrm{m} / \mathrm{z}$ [M+H $\mathrm{H}^{+}$, Calcd. for $\mathrm{C}_{24} \mathrm{H}_{34} \mathrm{~N}_{2} \mathrm{O}_{6} \mathrm{P}: 477.2155$, found 477.2165.

Diethyl (N-benzylamino)-3-piperidin-1-yl-(carboxymethyl)benzylphosphonate (6b) was obtained as a dense lightly yellow oil after flash chromatography using mixture of $\mathrm{CHCl}_{3} / \mathrm{MeOH}(15: 1)$ as eluent, ${ }^{31} \mathrm{P} \mathrm{NMR}\left(\mathrm{CHCl}_{3}\right.$, $121 \mathrm{MHz}$ ): $\delta_{\mathrm{P}} 23,05$ and 23,19. ${ }^{1} \mathrm{H} \mathrm{NMR}\left(\mathrm{CDCl}_{3}, 600 \mathrm{MHz}\right.$ ): $\delta_{\mathrm{H}} 1.10$ and 1.18 and 1,28 and 1.32 (each : t, J 7.0 $\mathrm{Hz}, 3 \mathrm{H}, \mathrm{OCH}_{2} \mathrm{CH}_{3}$ ), 1.53 (bs, $4 \mathrm{H}, \mathrm{CH}_{2} \mathrm{CH}_{2} \mathrm{NCH}_{2} \mathrm{CH}_{2}$ ), 1.82 (bs, 2H, $\mathrm{CH}_{2} \mathrm{CH}_{2} \mathrm{CH}_{2} \mathrm{~N}$ ), 3.15 (bs, 4H, $\mathrm{CH}_{2} \mathrm{NHCH}_{2}$ ), 3.54 and 3.57 (each : d, $\left.J_{\mathrm{HH}} 18,0 \mathrm{~Hz}, 2 \mathrm{H}, \mathrm{NHCH}_{2}\right), 3.79-4.19\left(\mathrm{~m}, 5 \mathrm{H}, \mathrm{OCH}_{2} \mathrm{CH}_{3}\right.$ and, $\mathrm{CHP}$ ), 4.72 and 4.74 (each: $\mathrm{s}, 1 \mathrm{H}$, $\mathrm{CHCOOH}), 7.25-7.29(\mathrm{~m}, 7 \mathrm{H}, \mathrm{Ar}), 7.31-7.32(\mathrm{~m}, 3 \mathrm{H}, \mathrm{Ar}), 7.37-7.43(\mathrm{~m}, 3 \mathrm{H}, \mathrm{Ar}), 7.52\left(\mathrm{~d}, \mathrm{~J}_{\mathrm{HH}} 6.0 \mathrm{~Hz}, 1 \mathrm{H}, \mathrm{Ar}\right), 7.59$ (d, $J_{\mathrm{HH}} 12.0 \mathrm{~Hz}, 1 \mathrm{H}, \mathrm{Ar}$ ), 7.61 (s, $\left.1 \mathrm{H}, \mathrm{Ar}\right), 7.65$ (d, $\mathrm{J}_{\mathrm{HH}} 12.0 \mathrm{~Hz}, 1 \mathrm{H}, \mathrm{Ar}$ ), 7.76 (s, 1H, Ar). $\left.{ }^{13} \mathrm{C} \mathrm{NMR} \mathrm{CDCl}_{3}, 151 \mathrm{MHz}\right)$ : $\delta_{\mathrm{C}}$ 16.27-16.37 (m, OCH $\left.\mathrm{CH}_{3}\right), 16.50\left(\mathrm{t}, J_{\mathrm{CP}} 4.5 \mathrm{~Hz}, \mathrm{OCH}_{2} \mathrm{CH}_{3}\right), 22.30\left(\mathrm{~d}, J_{\mathrm{CP}} 3.0 \mathrm{~Hz}, \mathrm{NCH}_{2} \mathrm{CH}_{2} \mathrm{CH}_{2}\right), 22.62(\mathrm{~s}$,

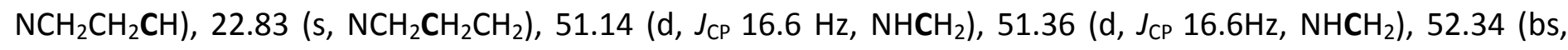
$\mathrm{CH}_{2} \mathrm{NCH}_{2}$ ), 59.09 (d, $\left.J_{\mathrm{CP}} 152.5 \mathrm{~Hz}, \mathrm{CHP}\right), 59.35$ (d, $\left.J_{\mathrm{CP}} 154.0 \mathrm{~Hz}, \mathrm{CHP}\right), 62.78$ (d, J JP $\left.6.0 \mathrm{~Hz}, \mathrm{OCH}_{2} \mathrm{CH}_{3}\right), 62.99\left(\mathrm{~d}, J_{\mathrm{CP}}\right.$ $7.6 \mathrm{~Hz}, \mathrm{OCH}_{2} \mathrm{CH}_{3}$ ), 63.25 (t, $J_{\mathrm{CP}} 6.0 \mathrm{~Hz}, \mathrm{OCH}_{2} \mathrm{CH}_{3}$ ), 74.77 and 74.87 (each : s, CH), 127.11 (s, Ar), 127.18 (s, Ar), 128.26-128.43 (m, Ar), 128.92 (s, Ar), 129.08 (s, Ar), 129.39 and 129.92 (each: d, J JP $6.0 \mathrm{~Hz}, \mathrm{Ar}$ ), 130.73 (bs, Ar), 132.42 (bs, Ar), 13265 (bs, Ar), 136.76 (d, Jc.P 4.5Hz, Ar), 136.92 (s, Ar), 139.17, (s, Ar), 139.25 (s, Ar), 169.07 (s, COOH). HRMS (ESI-TOF) m/z [M+H'], Calcd. for $\mathrm{C}_{24} \mathrm{H}_{34} \mathrm{~N}_{2} \mathrm{O}_{6} \mathrm{P}: 475.2362$, found 475.2352. 
Pinacolate of diethyl $\boldsymbol{\alpha}$-( $\boldsymbol{N}$-benzylamino)-4-boronobenzylphosphonate (7) 4-formylboronic acid (1.1 g, 7.3 $\mathrm{mmol}$ ) and pinacol $(0.9 \mathrm{~g}, 7.3 \mathrm{mmol})$ were dissolved in acetone $(40 \mathrm{~mL})$ and left at room temperature for $2 \mathrm{~h}$. Evaporation of the solvent produced light beige solid. It was dissolved in toluene $(40 \mathrm{~mL})$ and benzylamine $(0.84 \mathrm{~mL}, 7.7 \mathrm{mmole})$ was added and the mixture was refluxed for $1.5 \mathrm{~h}$ for azeotropic removal of formed water. To the obtained solution diethyl phosphite $(0.97 \mathrm{~mL}, 7.6 \mathrm{mmole})$ was then added and refluxing continued for $3 \mathrm{~h}$. After evaporation of solvent crude oil was chromatographically purified using hexane/ $\mathrm{CHCl}_{3}$ gradient (from $95 \%$ to $85 \%$ of hexane) as eluent yielding yellow solidifying oil (1.74 g, 52\%). ${ }^{31} \mathrm{P} \mathrm{NMR} \mathrm{(CDCl}{ }_{3}$,

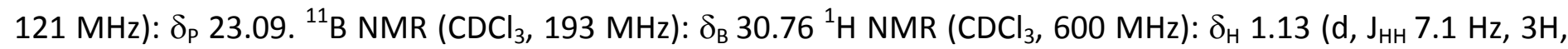
$\left.\mathrm{OCH}_{2} \mathrm{CH}_{3}\right), 1.29-1.31\left(\mathrm{~m}, 15 \mathrm{H}\right.$, rest of $\left.\mathrm{CH}_{3}\right), 3.66-3.73$ and 3.92-3.99 (each: $\left.\mathrm{m}, 1 \mathrm{H}, \mathrm{OCH}_{2} \mathrm{CH}_{3}\right), 4.07-4.19(\mathrm{~m}, 2 \mathrm{H}$, $\mathrm{OCH}_{2} \mathrm{CH}_{3}$ ), 4.84 (d, $\left.J_{\mathrm{PH}} 24.5 \mathrm{~Hz}, 1 \mathrm{H}, \mathrm{CHP}\right), 6.61$ and 7.59 (each: d, $J_{H H} 8.6 \mathrm{~Hz}, 2 \mathrm{H}, \mathrm{Ar}$ ), 7.26-7.29 (m, 1H, Ar), 7.34 $\left(\mathrm{t}, J_{\mathrm{HH}} 7.6 \mathrm{~Hz}, 2 \mathrm{H}, \mathrm{Ar}\right), 7.47-7.49(\mathrm{~m}, 2 \mathrm{H}, \mathrm{Ar}) .{ }^{13} \mathrm{C} \mathrm{NMR}\left(\mathrm{CDCl}_{3}, 151 \mathrm{MHz}\right): \delta_{\mathrm{C}} 16.20$ and 16.44 (each : $\mathrm{d}, J_{\mathrm{CP}} 5.6 \mathrm{~Hz}$, $\left.\mathrm{OCH}_{2} \mathrm{HCH}_{3}\right), 24.79$ and 24.85 each: s, $\left.\mathrm{BCCH}_{3}\right), 55.53\left(\mathrm{~d}, J_{\mathrm{CP}} 150.9 \mathrm{~Hz}, \mathrm{CHP}\right), 63.31$ and $63.38\left(\mathrm{~d}, J_{\mathrm{CP}} 6.5\right.$ $\mathrm{Hz}, \mathrm{OCH}_{2} \mathrm{CH}_{3}$ ), 83.25 (s, Ar), 112.96 (s, Ar), 127.83 (d, $J_{\mathrm{CP}} 5.5 \mathrm{~Hz}, \mathrm{Ar}$ ), 127.98 (d, J JP $3.0 \mathrm{~Hz}, \mathrm{Ar}$ ), 128.62 (d, J JP 2.0 $\mathrm{Hz}, \operatorname{Ar}$ ), 135.63 (s, Ar), 148.87 (d, J $J_{C P} 14.2 \mathrm{~Hz}, \mathrm{Ar}$ ). HRMS (ESI-TOF) m/z [M+H $\mathrm{H}^{+}$, Calcd. for $\mathrm{C}_{24} \mathrm{H}_{34} \mathrm{~N}_{2} \mathrm{O}_{6} \mathrm{P}$ : 460.2424 , found 460.2437 .

\section{Acknowledgements}

This work was supported by statuary activity subsidy from the Polish Ministry of Science and Higher Education.

\section{References}

1. Mucha, A.; Kafarski, P.; Berlicki, Ł. J. Med. Chem. 2011, 54, 5955.

http://dx.doi.org./10.1021/jm200587f

2. Orsini, F.; Sello, G; Sisti, M. Curr. Med. Chem. 2010, 17, 264.

http://dx.doi.org./10.2174/092986710790149729

3. Lejczak, B.; Kafarski, P. Top. Heterocycl. Chem. 2009, 20, 31. http://dx.doi.org./10.1007/7081 2008 14

4. Virieux, D.; Volle, J.-N.; Bakalara, N.; Pirat, J.-L. Top. Curr. Chem. 2015, 360, 39.

http://dx.doi.org./10.1007/128_2014_566

5. Naydenova, E. D.; Todorov P. T.; Troiev, K. D. Amino Acids. 2010, 38, 23.

http://dx.doi.org./10.1007/s00726-009-0254-7

6. Gałęzowska, J.; Gumienna-Kontecka, E.; Coord. Chem. Rev.. 2006, 265, 105.

http://dx.doi.org./10.1016/j.ccr.2011.07.002

7. Tarik, E. A.; Arkivoc 2014, (i), 21.

http://dx.doi.org./10.3998/ark.5550190.p008.189

8. Turcheniuk, K. V; Kukhar, V. P.; Röschenthaler, G.-V.; Aceña, J. L.; Soloshonok, V. A.; Sorochinsky, A. E. RSC Adv. 2013, 3, 6693.

http://dx.doi.org./10.1002/chem.201600758

9. Ordonez, M.; Viveros-Ceballos, J. L.; Cativiela, C.; Arizpe, A. Curr. Org. Synth. 2012, 9, 310. http://dx.doi.org./10.2174/157017912801270595

10. Kudzin, Z. H.; Kudzin, M. H.; Drabowicz, J.; Stevens, C. V.; Curr. Org. Chem. 2011, 15, 2015. http://dx.doi.org./10.2174/138527211795703612

11. Mynarz, P.; Rydzewska, A.; Pokładek, Z. J. Organomet. Chem. 2011, 696, 457. 
http://dx.doi.org./10.1016/j.jorganchem.2010.09.00

12. Piergies, N.; Proniewicz, E..; Kudelski, A. Rydzewska, A.; Kim, Y., Andrzejak, M.; Proniewicz, L. M. J. Phys. Chem. A 2012, 116, 10004.

http://dx.doi.org./10.1021/jp307064p

13. Petasis, N. A. Multicomponent reactions with organoboron compounds in: Multicomponent Reactions, Ed. Zhu, J.; Bienaymé, H. eds. 2005, Viley-VCH, pp. 199-223. http://dx.doi.org./10.1002/3527605118.ch7

14. Biggs-Houck, J. E.; Younai, A.; Shaw, J. T. Chem. Biol. 2010, 14, 371. http://dx.doi.org./10.1016/i.cbpa.2010.03.003

15. Yu, T.; Li, H.;.Wu, X., Yang, J. Chin. J. Org. Chem. 2012, 32, 1836. http://dx.doi.org./10.1002/cjoc.201090340

16. Shevchuk, M. V.; Sorochinsky, A. E.; Khilya, V. P.; Romanenko, V. D.; Kukhar, V. P. Synlett 2010, 1, 73. http://dx.doi.org./10.1055/s-0029-1218386 